\title{
Diagnostic difficile: entre choc et soulagement
}

\author{
Ursula Steiner-König
}

Dr, déléguée de la FMH au Comité central de la Journée des Malades

\section{Rétrospective}

Les malades concernés sont-ils les seuls à être sous le choc? Les médecins traitants ne le sont-ils pas aussi (parfois)? Je me souviens des cours donnés dans les années 70 par l'oncologue Gerd Nagel aux étudiants en médecine bâlois sur les maladies incurables et leur annonce au malade («Unheil-kunde»). Une approche révolutionnaire à l'époque tant, pendant longtemps, il était inconcevable de dire la vérité aux patientes et patients, surtout lorsqu'il s'agissait de diagnostics à l'issue fatale. Les diagnostics de cancer étaient ceux qui étaient les plus redoutés. Le corps médical estimait que le commun des mortels n'était pas capable d'affronter l'inéluctable fin de vie. On peut aujourd'hui retourner la question et se demander qui, des malades ou des médecins, avait finalement le plus de difficultés à aborder cette question? Quelques années plus tard, la menace des infections au virus du SIDA, qui plaçait la médecine face à une situation d'impuissance, allait entraîner des situations bien plus difficiles encore. Depuis, la réflexion autour de cette question a évolué. Dans le sillage de E. Kübler Ross, auteure de l'ouvrage «Les derniers instants de la vie» («On Death And Dying», paru en 1969), les publications sur l'accompagnement en fin de vie se sont multipliées. Un des enseignements essentiels de cette pionnière suisse de la recherche dans ce domaine consiste à dire que la mort relève de l'existence et de l'histoire personnelle de chaque individu. La société ne devrait pas fuir les questions sur la pertinence du principe du prolongement de la vie à tout prix.

Parallèlement, face à l'augmentation croissante des coûts de la santé, le débat sur le rationnement et la rationalisation des soins a pris de l'ampleur.

Enfin, la montée en puissance de l'internet a ouvert des possibilités jusque-là insoupçonnées d'accéder à des informations sur les symptômes, le diagnostic et les possibilités de traitement d'une maladie, sans passer par un interlocuteur médical.

\section{La situation actuelle}

Dans un contexte d'amélioration de la qualité et de rationalisation des méthodes de traitements médicaux, l'accent est désormais placé sur les diagnostics, les schémas thérapeutiques et les protocoles de soin; les économistes de la santé et les assureurs-maladie mettent au premier plan les critères d'économicité mais aussi d'éventuelles limitations aux soins. Le dialogue entre soignant et malade a tendance à se réduire aux pathologies en tant que telles. Forts des possibilités de l'internet, les patientes et patients ont tendance à s'improviser spécialistes du diagnostic. Et ils ne sont pas loin de revendiquer leurs compétences en matière de traitement avec un risque accru de surestimation de leurs capacités ou d'attentes démesurées. Ce n'est pas pour rien que l'on parle de plus en plus de consommateurs et non pas de patients. En outre, avec la généralisation du (Tiers garant, nous courons le risque de voir l'assureur-maladie s'immiscer toujours plus dans le contrat de soins entre médecin et patient et de chercher à infléchir les choix à son avantage.

Mais dans ce champ de vision, que reste-t-il de l'être humain, de la personne atteinte d'une maladie? En situation de choc, même s'ils n'en ont pas toujours conscience, les malades ont besoin de bien plus que d'une discussion rationnelle.

Dans les hôpitaux, suite aux mesures d'économies d'une part et au diktat des DRG et des forfaits par cas d'autre part, il est devenu quasiment impossible de se poser la question de savoir si la relation entre les patients et les médecins est suffisamment prise en compte. Le soignant pour sa part a besoin de faire une distinction: il va par exemple se demander si le malade est encore un patient en soins aigus ou s'il relève plutôt d'une unité de réadaptation ou de soins; s'il a besoin d'un psychiatre, d'un psychologue ou d'un travailleur social pour répondre à ses besoins d'information; ou encore déterminer qui doit s'occuper des proches et de leurs besoins? Qu'est-ce qui relève de la compétence des infirmières, de la physiothérapie, de l'ergothérapie ou d'autres catégories professionnelles?

\section{Perspectives}

Face à l'évolution des pathologies, les domaines traitant les formes de démence, les maladies chroniques 
ou la médecine palliative doivent être réévalués compte tenu d'une demande qui ne cesse de croître. L'accompagnement de malades incurables mobilise beaucoup d'actifs dans le domaine de la santé. Les soins palliatifs et - nouveau champ d'action - les soins spirituels sont appelés à répondre à des besoins souvent négligés des malades. La demande en personnel formé dans ce domaine s'accroît. On peut craindre un nouveau fractionnement des compétences ou, au contraire, voir dans cette évolution un nouveau défi. Il existe probablement un lien organique entre l'augmentation de la technicisation de la médecine d'une part et le besoin d'une dimension supplémentaire porteuse de sens d'autre part. Si nous, médecins, considérons cette dimension comme partie de notre mission, dans la pratique, il n'y a pas lieu de faire une séparation entre deux niveaux en théorie différents. La manière dont un médecin comprend sa mission s'oppose au morcellement de sa propre image.

A partir du printemps prochain, l'Université de Bâle proposera un nouveau master en soins spirituels (Advanced Studies, en formation continue) qui «enseigne au médecin à impliquer la dimension spirituelle dans l'approche de la maladie et de la santé, de la fin de vie et de la mort» (voir www.uniweiterbildung.ch)

Les propos ci-dessus nous révèlent que les «diagnostics difficiles" peuvent engendrer une situation de choc aussi du côté des soignants. Pour pouvoir apporter son aide à un patient, il faut avoir bénéficié d'une auto-assistance préalable permettant d'intégrer sa propre acceptation des limites de la vie et de développer une conception de la médecine comme l'art de soigner plutôt que de réparer. Une telle approche permettrait de s'affranchir d'attentes démesurées aussi bien envers soi-même qu'envers les patients qui nous sont confiés.

\section{Que faut-il au moment de la confronta- tion du médecin avec le patient concerné?}

Connaissons-nous suffisamment la personne? Dans la pratique, cet aspect se cristallise autour de différents éléments tels que l'âge de la / du malade, la durée du traitement et le genre de pathologies évoquées. Face aux limitations du temps disponible justifiées pour différentes raisons, il est légitime de se poser la question de savoir si jusqu'à présent, on a traité la personne ou les maladies. Je rapporterais les propos d'un collègue à ce sujet: «Ce médecin était un gestionnaire horspair de la maladie, mais ..." Manifestement, il lui a manqué de sensibilité, d'empathie et de présence dans le cas d'une patiente face à un diagnostic oncologique particulièrement difficile.

Suis-je en mesure de voir la situation en face? En fonction de nos expériences de vie, que ce soit dans le cercle familial ou des proches ou dans la pratique au cabinet, et aussi en fonction de notre âge, nous abordons les questions des limites de la vie de diverses manières. La frayeur des débuts laisse progressivement la place à une plus grande aisance face à cette question.

Comment vais-je accueillir les réactions du malade? L'incertitude liée à cette question représente la plus grande tentation de vouloir éluder tout échange en profondeur avec la personne malade. A moins d'arriver à cacher ses propres émotions.

Qu'en est-il de ses propres compétences? L'importance de cet aspect dépend assurément de la spécialisation: en oncologie, on aura un comportement différent qu'en orthopédie. Aucune discipline n'est cependant épargnée et ce ne sont pas les médecins de famille qui diront le contraire. Cependant, plus nous sommes régulièrement confrontés à des situations bouleversantes, moins nous en sommes affectés. De même, dans la communication, il y aura forcément une différence s'il s'agit de l'annonce d'une maladie chronique ou d'une question de vie ou de mort, avec des conséquences massives sur la vie quotidienne. Même si une maladie chronique peut aussi durablement affecter la manière de vivre ou les perspectives d'avenir d'une personne, l'espérance d'un sens à donner à son existence peut plus facilement reprendre le dessus. La médecine comme art de soigner peut alors s'affirmer dans la durée pour le bien des patientes et patients.

\section{Post-scriptum}

Durant la rédaction du présent article sur la Journée des Malades, notre Bulletin des médecins suisses a publié un document de la DDQ intitulé «Shared Decision Making - Médecins et patients décident ensemble» [1]. Suite à cette publication, je renonce à développer davantage la question pour ne pas «porter de l'eau à la rivière». En revanche, je félicite notre FMH et en particulier les auteurs de l'article pour sa parution.

1 Gerber M, Kraft E, Bosshard C. Shared Decision Making - Médecins et patients décident ensemble. Bull Méd Suisses. 2014;95(50):1883-9. 